\title{
Arreglos musicales en el aula: Factores pedagógicos en la Educación Primaria
}

\section{Music Arrangement in the Classroom: Teaching Factors in Primary Education}

\author{
Jean Huillipan Rain \\ jeanhuillipan@gmail.com \\ Instituto de Música \\ Universidad Alberto Hurtado \\ Santiago, Chile \\ ORCID: https://orcid.org/0000-0002-1800-2667 \\ Rolando Ángel-Alvarado \\ rolando.angel.alvarado@gmail.com \\ Instituto de Música \\ Universidad Alberto Hurtado \\ Santiago, Chile \\ ORCID: https://orcid.org/0000-0002-1800-2667
}

doi: 10.7203/LEEME.45.16527

Recibido: 17-01-2020 Aceptado: 15-03-2020. Contacto y correspondencia: Rolando Ángel-Alvarado, Instituto de Música Universidad Alberto Hurtado, Calle Almirante Barroso 31, C.P. 8340540 Santiago. Chile.

\section{Resumen}

El objetivo del presente estudio es identificar factores pedagógicos que influyen al montar arreglos musicales en el aula regular de Educación Primaria, dado que existe la posibilidad que el profesorado tenga dificultades para equilibrar los fines musicales con los pedagógicos al elaborar y montar arreglos musicales. La investigación tiene un diseño cualitativo, no experimental y transeccional, utilizándose el método comparativo constante de orden inductivo para recoger y analizar los datos. La muestra de tipo deliberada se compone de dos docentes especialistas en educación musical de amplia trayectoria en los niveles de Educación Primaria. Los resultados indican que el dominio de las competencias musicales y pedagógicas son determinantes para resolver problemas didácticos concernientes a la creación y montaje de arreglos musicales. Al concluir, se han identificado tres factores pedagógicos que inciden en contextos de Educación Primaria: la autonomía del docente, la estructura de la clase y, finalmente, la estructura misma del arreglo.

Palabras clave: Educación obligatoria; educación musical; prácticas educativas; experiencia pedagógica.

\section{Abstract}

This study aims to identify teaching factors that influence the implementation of music arrangements in the classroom of primary education, given that educators can face difficulties balancing musical and teaching aspects at the moment of elaborating and implementing music arrangements. This research holds a qualitative, nonexperimental, and cross-sectional design, utilising it the constant comparative method in an inductive order for collecting and analysing data. The purposive sample is integrated by two teachers specializing in music education who have a vast experience in primary schools. Results display that control of musical and pedagogical competencies are essential for solving teaching problems linked to the creation and implementation of music arrangements. In conclusion, three teaching factors have identified in milieus of primary education: teacher autonomy, lesson structure, and musical structure of the arrangement.

Key words: Compulsory education; music education; educational practices; teaching experience.

@ Jean Huillipan Rain y Rolando Ángel-Alvarado. The content of this article is the sole responsibility of the authors. The Revista Electrónica de LEEME and Universitat de València are not liable for any legal actions that may arise involving the article's content. Revista Electrónica de LEEME - Lista Electrónica Europea de Música en la Educación-. http://ojs.uv.es/index/php/LEEME/index ISSN: 1575-9563. Editores: Universidad de Valencia y Jesús Tejada. Visibilidad de esta revista: SCOPUS, Emerging Sources Citation Index (Clarivate), EBSCO, CINDOC (CSIC), Citefactor, COPAC, Dialnet, DICE (CSIC), DOAJ, e-revistas (CSIC), EBSCO Premier, ERIH+, Gale Cengage Learning, IN-RECS, IRESIE, LATINDEX, MIAR, OCLC Worldcat, RESH, REDIB, RILM Core Journals, SUDOC, ULRICHS. Esta revista es de acceso libre mediante licencia Creative Commons 4.0 CC by. Política de archivado: etiqueta verde SHERPA-ROMEO. 


\section{Introducción}

Este estudio propone aportar en el ámbito pedagógico de la creación y montaje de obras musicales (Silva, 2019), puesto que el profesorado tendría dificultades para articular y equilibrar los fines musicales con los pedagógicos (Ángel-Alvarado, 2018) al momento de elaborar arreglos, lo que podría desencadenar procesos de aprendizaje poco significativos debido a la utilización de partituras mal instrumentadas o armonizadas. Esta situación deja entrever que la calidad educativa se define, en cierta parte, por las competencias educativo-musicales del profesorado (López-Iñiguez y Pozo, 2014b), dado que los arreglos musicales actúan como material pedagógico en los procesos de aprendizaje y desarrollo instrumental que experimenta cada estudiante (López-Iñiguez y Pozo, 2014a).

En tal sentido, el Ministerio de Educación de Chile (MINEDUC, de ahora en adelante) recomienda entregar las partituras al estudiantado con el afán de promover las prácticas instrumentales (MINEDUC, 2013), poniendo el foco en favorecer el desarrollo real del alumnado. En el marco de dicha intención, el currículo nacional para la asignatura de Música dirigido a la Educación Primaria (MINEDUC, 2013) establece objetivos y orientaciones pedagógicas ligadas al cantar y tocar instrumentos musicales, aportando una serie de arreglos y repertorios que pueden emplearse a libre disposición, los que pueden incluso modificarse o mejorarse (Cantón y Vargas 2014) según el criterio del profesorado. Al tratarse de un currículo abierto (Ángel-Alvarado, 2018), el profesorado es libre de utilizar otros repertorios que no se incluyen en el currículo nacional.

Por todo lo anterior, es plausible aseverar que diversos estilos de enseñanza se ponen en práctica en el sistema educativo con el fin de favorecer el aprendizaje musical (Elliott y Silverman, 2015; Kertz-Welzel, 2018; Odendaal, Kankkunen, Nikkanen, y Vakeva, 2014). Esta diversidad de estilos pedagógicos saca a la luz la existencia de una multiplicidad de representaciones acerca de la teleología de la educación musical (Ángel-Alvarado, 2020), ya que un grupo de docentes priorizaría el desempeño técnico performativo (Carey, Harrison y Dwyer, 2017; Salvador, 2019), otro pondría el foco en la potenciación creativa (ÁngelAlvarado, Wilhelmi y Belletich, 2019b), un tercer grupo podría centrarse principalmente en la lecto-escritura musical (Fautley, 2017) y, así, otros sectores del magisterio podrían priorizar otras áreas de desarrollo musical o integral. En consecuencia, queda en evidencia la falta de consenso profesional respecto a los fines o propósitos que tiene la educación musical en el currículo de Educación Primaria, siendo esto el principal motivo por el que se necesitan identificar factores pedagógicos determinantes a la hora de emprender proyectos de creación y montaje de arreglos musicales.

@ Jean Huillipan Rain y Rolando Ángel-Alvarado. The content of this article is the sole responsibility of the authors. The Revista Electrónica de LEEME and Universitat de València are not liable for any legal actions that may arise involving the article's content. Revista Electrónica de LEEME - Lista Electrónica Europea de Música en la Educación-. http://ojs.uv.es/index/php/LEEME/index ISSN: 1575-9563. Editores: Universidad de Valencia y Jesús Tejada. Visibilidad de esta revista: SCOPUS, Emerging Sources Citation Index (Clarivate), EBSCO, CINDOC (CSIC), Citefactor, COPAC, Dialnet, DICE (CSIC), DOAJ, e-revistas (CSIC), EBSCO Premier, ERIH+, Gale Cengage Learning, IN-RECS, IRESIE, LATINDEX, MIAR, OCLC Worldcat, RESH, REDIB, RILM Core Journals, SUDOC, ULRICHS. Esta revista es de acceso libre mediante licencia Creative Commons 4.0 CC by. Política de archivado: etiqueta verde SHERPA-ROMEO. 
Por consiguiente, el presente estudio establece el objetivo de identificar factores pedagógicos que influyen al montar arreglos musicales en el aula regular de Educación Primaria. Entonces, es preciso comprender que, para efectos de este estudio, los factores pedagógicos hacen referencia a decisiones docentes que apuntan a potenciar el desempeño estudiantil en las actividades ligadas a arreglos musicales, mientras que el aula regular se entiende como la congregación de un grupo de estudiantes que asiste a la clase de Música por obligación curricular. Dicho esto, cabe señalar que para alcanzar el objetivo se han recogido datos de dos docentes de educación musical que ejercen en la zona céntrica de Santiago de Chile (Chile), quienes cuentan con más de 10 años de experiencia pedagógica en aulas de Educación Primaria. Aquellos sujetos han participado en entrevistas semiestructuradas, así como también han sometido un arreglo de su autoría a evaluación.

\section{Método}

Este estudio cualitativo, no-experimental y transeccional se enmarca en el método comparativo constante de orden inductivo, puesto que la codificación de los datos se organiza a partir de la "subjetividad inductiva del investigador" (Bonilla-García y López-Suárez, 2016, p.308) para clasificar e interrelacionar los códigos que emergen directamente desde los datos que proveen los informantes. La saturación de los datos dará lugar a una categoría central (San Martín, 2014), que se podrá entender como una teoría emergente en el marco de los factores pedagógicos que influyen al abordar arreglos musicales en el aula regular de Educación Primaria. En cambio, la subjetividad inductiva se forja desde dos categorías conceptuales que hacen factible los procesos de clasificación, interrelación y selección de códigos. En concreto, estas categorías conceptuales son:

1. Repertorios propuestos por el currículo nacional para la asignatura de Música.

2. El arreglo como estrategia didáctica.

\subsection{Contexto}

En Chile, a pesar de que el currículo nacional busque una entrega equitativa de los contenidos en las escuelas mediante la propuesta de arreglos y repertorios, la equidad no es posible dada la diversidad de realidades en los centros educativos existentes en el país (Muñoz, López y Assaél, 2015). Las escuelas participantes en el presente estudio sirven de ejemplo.

Una de ellas es un centro educativo privado que cuenta con las condiciones de infraestructura necesarias para la labor musical. Por ejemplo, hay dos aulas de Música equipadas con altavoces (parlantes), una mesa de sonido y un reproductor musical, más todo el equipo técnico audiovisual (proyector, computador y pantalla de proyección o telón). En cuanto al equipamiento instrumental, poseen guitarras acústicas, guitarras y bajos eléctricos con sus

@ Jean Huillipan Rain y Rolando Ángel-Alvarado. The content of this article is the sole responsibility of the authors. The Revista Electrónica de LEEME and Universitat de València are not liable for any legal actions that may arise involving the article's content. Revista Electrónica de LEEME - Lista Electrónica Europea de Música en la Educación-. http://ojs.uv.es/index/php/LEEME/index ISSN: 1575-9563. Editores: Universidad de Valencia y Jesús Tejada. Visibilidad de esta revista: SCOPUS, Emerging Sources Citation Index (Clarivate), EBSCO, CINDOC (CSIC), Citefactor, COPAC, Dialnet, DICE (CSIC), DOAJ, e-revistas (CSIC), EBSCO Premier, ERIH+, Gale Cengage Learning, IN-RECS, IRESIE, LATINDEX, MIAR, OCLC Worldcat, RESH, REDIB, RILM Core Journals, SUDOC, ULRICHS. Esta revista es de acceso libre mediante licencia Creative Commons 4.0 CC by. Política de archivado: etiqueta verde SHERPA-ROMEO. 
respectivos amplificadores, teclados electrónicos, set de percusiones menores latinoamericanas, tres baterías acústicas y una electrónica, glockenspiels, flautas dulces, micrófonos con sus atriles, más atriles de partitura. En términos administrativos, dicha escuela tiene un Departamento de Música que se compone de cuatro docentes, lo que permite articular diferentes proyectos musicales. Para la clase de Música, todo el estudiantado lleva sus propios instrumentos y dentro de esas opciones están: guitarras, ukeleles, glockenspiels, melódicas, teclados electrónicos y flautas dulces.

La otra escuela es pública, dependiendo administrativamente de la Municipalidad de Santiago Centro. Dicha escuela no cuenta con un aula de Música, la cantidad de instrumentos es precaria, ya que únicamente se observan algunas guitarras, flautas dulces, 30 glockenspiels, un set de percusiones menores latinoamericanas y dos teclados. Para poder realizar las clases, el docente debe trasladar a cada aula los instrumentos ya indicados, de modo que ha designado a un grupo de estudiantes la labor de ayudarle con el transporte, con el ánimo de agilizar la labor de traslado y, así, disponer de mayor cantidad de tiempo para trabajar en clase. Cabe señalar que la escuela registra una alta tasa de matrícula de estudiantes provenientes de otros países latinoamericanos.

\subsection{Muestra}

El tipo de muestra fue no probabilístico y deliberado, ya que las personas seleccionadas debían cumplir un determinado perfil para participar en el estudio. A saber, los dos docentes participantes tienen como mínimo 10 años de experiencia pedagógica en Educación Primaria, son docentes especialistas porque poseen oficialmente el título de Profesor de Música y, por último, se hacen cargo de confeccionar sus propios arreglos musicales. A pesar de lo anterior, es necesario indicar que existen diferencias entre ambos educadores. En concreto, el Docente 1 cuenta con un Diplomado en Terapias Corporales y Artísticas, así como también estudios certificados para ejercer en contexto de educación musical temprana. Además, se ha desempeñado como músico en diversas agrupaciones de música popular y obras de música infantil. Su labor docente la realiza precisamente desde los niveles preescolares hasta sexto básico (entendido en España como sexto de Primaria).

Y, el Docente 2 ha realizado su labor en diversos centros educativos, tanto del sector privado como público, contando con experiencia en todos los niveles educativos que comprende el currículo nacional: Preescolar, Primaria, Secundaria Inferior y Secundaria Superior. Cuenta además con un Máster en Dirección de Conjuntos Instrumentales y, por último, cabe señalar que en su faceta de músico se desempeña como baterista y percusionista clásico.

@ Jean Huillipan Rain y Rolando Ángel-Alvarado. The content of this article is the sole responsibility of the authors. The Revista Electrónica de LEEME and Universitat de València are not liable for any legal actions that may arise involving the article's content. Revista Electrónica de LEEME - Lista Electrónica Europea de Música en la Educación-. http://ojs.uv.es/index/php/LEEME/index ISSN: 1575-9563. Editores: Universidad de Valencia y Jesús Tejada. Visibilidad de esta revista: SCOPUS, Emerging Sources Citation Index (Clarivate), EBSCO, CINDOC (CSIC), Citefactor, COPAC, Dialnet, DICE (CSIC), DOAJ, e-revistas (CSIC), EBSCO Premier, ERIH+, Gale Cengage Learning, IN-RECS, IRESIE, LATINDEX, MIAR, OCLC Worldcat, RESH, REDIB, RILM Core Journals, SUDOC, ULRICHS. Esta revista es de acceso libre mediante licencia Creative Commons 4.0 CC by. Política de archivado: etiqueta verde SHERPA-ROMEO. 


\subsection{Instrumento}

Las dos técnicas utilizadas fueron validadas por un panel de expertos conformado por un compositor musical, dos docentes de educación musical, un editor de transcripciones musicales y una socióloga. Así, las técnicas pudieron afinarse tras recibir retroalimentaciones desde distintas disciplinas de las ciencias de la educación musical.

La primera técnica es una entrevista semiestructurada que se centra en identificar las estrategias pedagógicas que utiliza el profesorado para crear y montar arreglos musicales en contextos de Educación Primaria. Se compone de ocho preguntas distribuidas en tres criterios:

Criterio 1. Implementación del currículo nacional:

a. ¿Desarrolla las clases en base a las unidades propuestas por el MINEDUC en el currículum?

b. ¿Cree usted que el repertorio propuesto por el MINEDUC ayuda al desarrollo de las clases en el aula?

Criterio 2. El arreglo como estrategia didáctica:

a. ¿Cuáles son los criterios que utiliza al momento de elegir el repertorio para trabajar con el estudiantado en el aula?

b. Dentro del repertorio seleccionado para trabajar con el estudiantado. ¿Realiza efectivamente algún tipo de arreglo musical para la pieza? Si la respuesta es afirmativa, ¿Qué tipo de arreglo realiza? y ¿por qué motivo? (recursos materiales, infraestructura, estrategia didáctica o motivacional).

c. De acuerdo a su experiencia en el aula, al momento de implementar el arreglo musical como estrategia didáctica. ¿De qué forma se manifiestan los estudiantes frente a esto?

d. Según su experiencia docente. ¿Considera que existe material suficiente que oriente a la realización de arreglos musicales para un contexto escolar en Primer Ciclo Básico?

Criterio 3. Formación docente:

a. ¿Cree que las universidades que imparten pedagogía en educación musical debieran dar mayor importancia asignaturas que contribuyan a la elaboración de arreglos para el aula en todo contexto escolar?

b. ¿Considera que su formación académica superior significó un aporte concreto para desarrollarse en esta área?

La segunda técnica es una pauta de evaluación que pone el foco en identificar la idoneidad y pertinencia de los arreglos que elabora el profesorado en los siguientes aspectos: forma musical del arreglo, tipo de armonía, instrumentación, dificultad rítmica, cantidad de acordes, pertinencia didáctica de la tonalidad para aprender a ejecutar un instrumento, cantidad

@ Jean Huillipan Rain y Rolando Ángel-Alvarado. The content of this article is the sole responsibility of the authors. The Revista Electrónica de LEEME and Universitat de València are not liable for any legal actions that may arise involving the article's content. Revista Electrónica de LEEME - Lista Electrónica Europea de Música en la Educación-. http://ojs.uv.es/index/php/LEEME/index ISSN: 1575-9563. Editores: Universidad de Valencia y Jesús Tejada. Visibilidad de esta revista: SCOPUS, Emerging Sources Citation Index (Clarivate), EBSCO, CINDOC (CSIC), Citefactor, COPAC, Dialnet, DICE (CSIC), DOAJ, e-revistas (CSIC), EBSCO Premier, ERIH+, Gale Cengage Learning, IN-RECS, IRESIE, LATINDEX, MIAR, OCLC Worldcat, RESH, REDIB, RILM Core Journals, SUDOC, ULRICHS. Esta revista es de acceso libre mediante licencia Creative Commons 4.0 CC by. Política de archivado: etiqueta verde SHERPA-ROMEO. 
de melodías que participan de forma vocal o instrumental, rol docente durante el montaje del arreglo (solo dirección o apoya en la ejecución instrumental).

\subsection{Procedimientos de recogida de datos}

En primer lugar, se ha invitado a escuelas y a profesorado, en particular, mediante una carta, procediendo luego a la firma de la autorización institucional para realizar la recogida de datos en las escuelas, así como también a la firma de los consentimientos informados que dan garantía del aseguramiento de los códigos éticos de la actividad investigadora. Inmediatamente después, se procedió a fijar el día en que se llevaría a cabo la entrevista semiestructurada, con el fin de realizar la actividad dentro de la jornada laboral del profesorado participante. Asimismo, se establecieron acuerdos para coordinar la entrega del arreglo que sería evaluado en idoneidad y pertinencia didáctica, concretándose la entrega la primera quincena de octubre de 2019. En lo que respecta a la entrevista, fue realizada de forma presencial y privada durante la última semana de septiembre y la segunda semana de octubre en las escuelas en que el profesorado participante desempeña sus funciones académicas, siendo ambas entrevistas registradas en formato audio bajo el consentimiento del personal implicado.

\subsection{Procedimientos de análisis de datos}

A partir de las categorías conceptuales, se ha procedido a codificar los datos. En primer lugar, se realizó el proceso de codificación abierta, en donde se presentan los datos tal como se consiguieron (San Martín, 2014) en las categorías conceptuales presentadas anteriormente. De esta forma, se procede a la comparación y saturación de los mismos, dando cumplimiento al proceso de codificación axial. Finalmente, las interrelaciones se saturan, permitiendo seleccionar los códigos de mayor relevancia mediante el proceso de codificación selectiva. De este último proceso, se desprende la categoría central que permitirá dar cumplimiento al objetivo del estudio, brindando una explicación teórico-empírica (San Martín, 2014).

\section{Resultados}

\subsection{Repertorios propuestos por el currículo nacional para la asignatura de Música}

El profesorado participante expresa que el repertorio que sugiere el currículo nacional no es pertinente para la realidad educativa actual. Más precisamente, el Docente 1 señaló sentir "que el repertorio no está contextualizado a la realidad", mientras que el Docente 2 indicó "que la propuesta curricular es insatisfactoria y descontextualizada". Ambas razones dan cuenta de un repertorio antiguo que no genera interés en el profesorado, puesto que el Docente 1 ha manifestado que en el currículo nacional no encuentra "un repertorio considerable en cuanto a

@ Jean Huillipan Rain y Rolando Ángel-Alvarado. The content of this article is the sole responsibility of the authors. The Revista Electrónica de LEEME and Universitat de València are not liable for any legal actions that may arise involving the article's content. Revista Electrónica de LEEME - Lista Electrónica Europea de Música en la Educación-. http://ojs.uv.es/index/php/LEEME/index ISSN: 1575-9563. Editores: Universidad de Valencia y Jesús Tejada. Visibilidad de esta revista: SCOPUS, Emerging Sources Citation Index (Clarivate), EBSCO, CINDOC (CSIC), Citefactor, COPAC, Dialnet, DICE (CSIC), DOAJ, e-revistas (CSIC), EBSCO Premier, ERIH+, Gale Cengage Learning, IN-RECS, IRESIE, LATINDEX, MIAR, OCLC Worldcat, RESH, REDIB, RILM Core Journals, SUDOC, ULRICHS. Esta revista es de acceso libre mediante licencia Creative Commons 4.0 CC by. Política de archivado: etiqueta verde SHERPA-ROMEO. 
número que sea atractivo, no es algo que llame mucho la atención". Para revertir la situación, el Docente 1 reconoce utilizar repertorios que le motivan a él, poniendo énfasis en el siguiente discurso: "que me motive mucho a mí, algo que yo ya conozca, que me guste, yo creo que eso es clave". Además, el Docente 1 agrega que: "si es un repertorio que encuentro que a mí no me llama mucho..., lo más seguro es que naturalmente los chiquillos sientan eso".

La novedad musical también es un factor a considerar, dado que el Docente 2 hizo referencia a que hay canciones que aprendió en la universidad y que actualmente al estudiantado "no le entusiasma para nada". A esto, cabe agregar cuestiones ligadas a la educación inclusiva, ya que algunos repertorios han dejado de ser abordables. Por ejemplo:

Hay una canción que se llama El negrito aquel y no puede ser utilizada porque en un colegio con una cantidad importante de migrantes, pronto comienzan las bromas y las risas y eso por ahora debemos evitarlo al máximo, estamos en un proceso de cambio y de aceptación aún. (Docente 2)

No obstante, cabe señalar que a pesar de que el profesorado no toma en consideración el repertorio propuesto en el currículo nacional, sí utiliza la política educativa para dar lineamientos generales al trabajo docente en el aula (Figura 1). En concreto, el Docente 1 señaló que "cuando uno lee el propósito de cada unidad, entiende para dónde debe uno dirigir el trabajo, más que mirar los objetivos de aprendizaje, porque los objetivos de aprendizaje se van repitiendo durante cada unidad". Entonces, no es que el currículo nacional quede completamente desplazado, sino que son rescatadas otras sugerencias. Por ejemplo, el Docente 2 indica que ha "tomado las pautas de evaluación, rúbricas y todo eso que es impecable".
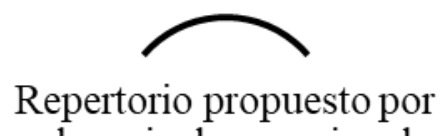

el curriculum nacional

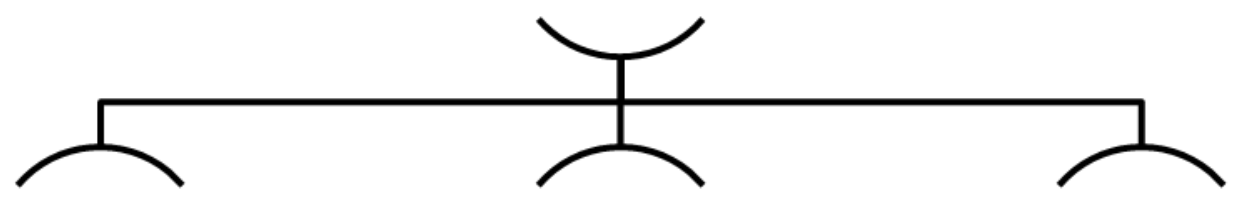

Descontextualización del repertorio

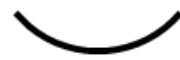

Cambios culturales en la sociedad

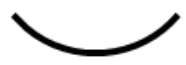

Sugerencias pedagógicas

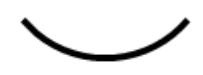

Figura 1. Códigos abiertos de la primera categoría conceptual Fuente: Elaboración propia

@ Jean Huillipan Rain y Rolando Ángel-Alvarado. The content of this article is the sole responsibility of the authors. The Revista Electrónica de LEEME and Universitat de València are not liable for any legal actions that may arise involving the article's content. Revista Electrónica de LEEME - Lista Electrónica Europea de Música en la Educación-. http://ojs.uv.es/index/php/LEEME/index ISSN: 1575-9563. Editores: Universidad de Valencia y Jesús Tejada. Visibilidad de esta revista: SCOPUS, Emerging Sources Citation Index (Clarivate), EBSCO, CINDOC (CSIC), Citefactor, COPAC, Dialnet, DICE (CSIC), DOAJ, e-revistas (CSIC), EBSCO Premier, ERIH+, Gale Cengage Learning, IN-RECS, IRESIE, LATINDEX, MIAR, OCLC Worldcat, RESH, REDIB, RILM Core Journals, SUDOC, ULRICHS. Esta revista es de acceso libre mediante licencia Creative Commons 4.0 CC by. Política de archivado: etiqueta verde SHERPA-ROMEO. 
En definitiva, es esencial que el profesorado elabore arreglos con la intención de estrechar lazos con el estudiantado. En este marco, el Docente 1 señaló que "uno va haciendo [arreglos] en base a lo que conoce del curso, de lo que tocan", lo que se refuerza con el discurso centrado en la necesidad de conocer "el contexto, las capacidades y niveles del estudiantado" (Docente 2). Por consiguiente, el profesorado considera que con el arreglo pueden abordar muchas de las actividades que propone el MINEDUC por medio del currículo nacional, a pesar de la descontextualización del repertorio. No obstante, hacen hincapié en la idea de que la clase de Música siempre tiene que sonar, tener un sentido y ser entretenida.

\begin{abstract}
Me interesa que lo que salga, el producto de la clase. Primero sea motivante para los niños y que no sea frustrante, me interesa que suene, que ellos sientan que lo que están haciendo está sonando. Entonces, cuando los niños se dan cuenta y las niñas se dan cuenta que esto suena, viven una experiencia musical gratificante. (Docente 1)
\end{abstract}

\title{
3.2 El arreglo como estrategia didáctica
}

El acto de elaborar y montar arreglos musicales nace de la premisa de que el profesorado también se desempeña como instrumentista, lo que queda en evidencia con el discurso del Docente 2, quien expresa: "Cuando sientes con propiedad esta idea de montar [arreglos], sigues ese camino... Uno toca porque uno es músico. Eso es lo que en el fondo uno hace". A partir de esta opinión, el profesorado se atreve a incentivar el uso de instrumentos musicales desde el primer nivel de Educación Primaria, potenciando así el aprendizaje y desarrollo de las competencias instrumentales tanto individuales como colectivas.

El arreglo no se aborda únicamente desde la partitura tradicional, puesto que en los primeros niveles de Educación Primaria se suelen utilizar recursos no convencionales, como, por ejemplo, símbolos, pictogramas y musicogramas. En este punto, el Docente 1 precisa que la utilización de la notación musical clásica no es importante para él, especialmente en los primeros niveles de Educación Primaria, ya que el alumnado se puede sentir frustrado "porque no sabe leer una partitura". Bajo ese pensamiento está alineado el trabajo de la realización del arreglo, donde el Docente 1 expresa: "eso me lleva hasta en cambiarles la métrica en algunas canciones como La Pincoya, que es una pericona", la que está originalmente en 12/8, pero la partitura (Figura 2) la presenta en 2/4.

A lo anterior, el Docente 2 agrega: "ahora, por ejemplo, si la canción tiene bemoles, tienes que adaptarla porque los metalófonos [glockenspiel] tienen todo con sostenidos". Por tanto, queda en evidencia la necesidad por tocar todo en tonalidades cómodas. Específicamente, el Docente 1 indica "que los niños toquen... en la escala de Do Mayor o de La Menor y que cuando vayan a cantar lo modulo, cosa que puedan cantar en el registro de ellos". En la misma línea, el Docente 2 menciona: "entonces tomo una melodía y le hago un acompañamiento. Ahí

@ Jean Huillipan Rain y Rolando Ángel-Alvarado. The content of this article is the sole responsibility of the authors. The Revista Electrónica de LEEME and Universitat de València are not liable for any legal actions that may arise involving the article's content. Revista Electrónica de LEEME - Lista Electrónica Europea de Música en la Educación-. http://ojs.uv.es/index/php/LEEME/index ISSN: 1575-9563. Editores: Universidad de Valencia y Jesús Tejada. Visibilidad de esta revista: SCOPUS, Emerging Sources Citation Index (Clarivate), EBSCO, CINDOC (CSIC), Citefactor, COPAC, Dialnet, DICE (CSIC), DOAJ, e-revistas (CSIC), EBSCO Premier, ERIH+, Gale Cengage Learning, IN-RECS, IRESIE, LATINDEX, MIAR, OCLC Worldcat, RESH, REDIB, RILM Core Journals, SUDOC, ULRICHS. Esta revista es de acceso libre mediante licencia Creative Commons 4.0 CC by. Política de archivado: etiqueta verde SHERPA-ROMEO. 
ocupo Mi Menor, Sol Mayor, La Menor etc. Melodías con acompañamientos en tonalidades súper sencillas".

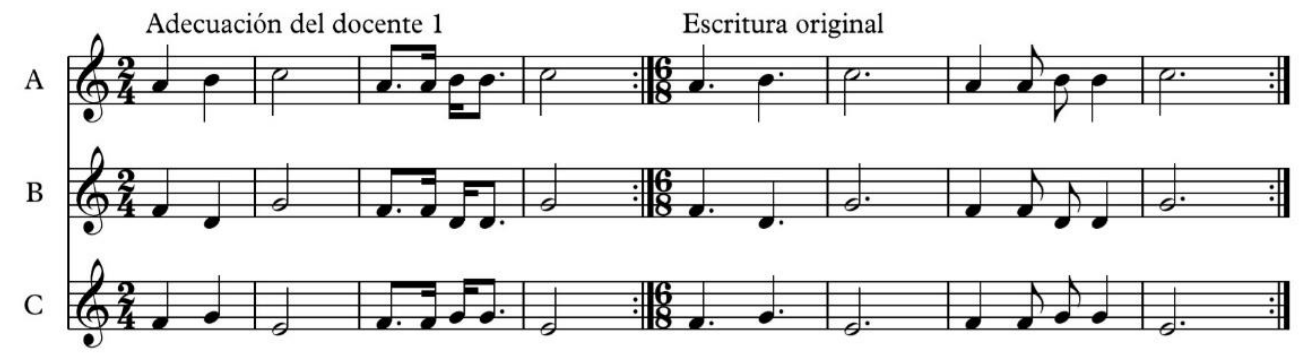

Figura 2. Introducción de "La Pincoya" Fuente: Elaboración propia

Con respecto a la variedad instrumental, el profesorado plantea el interés por aprovechar todos los recursos sonoros que enriquezcan timbrísticamente al arreglo. En concreto, el Docente 1 expresa:

Ocupo los distintos timbres instrumentales que tengo dentro de la sala de clases.... Si tenemos cuatro timbres instrumentales dentro de la sala de clase, hacerlos tocar todo al unísono no puede ser, estaríamos perdiendo algo que podría ser una riqueza. (Docente 1)

La estructura clara y sencilla del arreglo también propicia un buen desempeño musical del alumnado. Por ejemplo, el Docente 1 informa que, en los arreglos, como La Pincoya:

Generalmente le incorporo a las piezas una introducción que es distinta a la melodía general de la canción, no es que imitemos siempre la melodía que se canta en lo que se toca y esa misma introducción la ocupo como material para hacer un interludio o como coda, entonces los chicos van cantando y tocando. (Docente 1)

Así se logra articular la práctica instrumental con la vocal en el arreglo. El Docente 1 indica que "van haciendo un trabajo coral y un trabajo de conjunto instrumental en otros momentos, pero siempre dentro de la misma pieza".

$\mathrm{Si}$ bien todos los cambios se pueden realizan en lo teórico-práctico musical, hay elementos pedagógicos que permiten y refuerzan la estrategia y desarrollo del arreglo en el aula. El orden y la disciplina para abordar este trabajo es fundamental, la distribución en el aula ayuda a realizar dicha actividad. En concreto, el Docente 1 menciona la importancia de "armar una modalidad por tipo de instrumento. Las cuerdas en un lado, los vientos en otro, la percusión en otro, la percusión melódica en este sector, las teclas por tal lado". Dicha organización permitiría simular el trabajo de un director de orquesta.

@ Jean Huillipan Rain y Rolando Ángel-Alvarado. The content of this article is the sole responsibility of the authors. The Revista Electrónica de LEEME and Universitat de València are not liable for any legal actions that may arise involving the article's content. Revista Electrónica de LEEME - Lista Electrónica Europea de Música en la Educación-. http://ojs.uv.es/index/php/LEEME/index ISSN: 1575-9563. Editores: Universidad de Valencia y Jesús Tejada. Visibilidad de esta revista: SCOPUS, Emerging Sources Citation Index (Clarivate), EBSCO, CINDOC (CSIC), Citefactor, COPAC, Dialnet, DICE (CSIC), DOAJ, e-revistas (CSIC), EBSCO Premier, ERIH+, Gale Cengage Learning, IN-RECS, IRESIE, LATINDEX, MIAR, OCLC Worldcat, RESH, REDIB, RILM Core Journals, SUDOC, ULRICHS. Esta revista es de acceso libre mediante licencia Creative Commons $4.0 \mathrm{CC}$ by. Política de archivado: etiqueta verde SHERPA-ROMEO. 


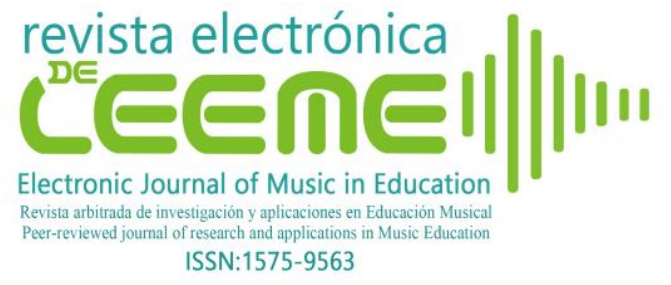

Así uno puede ir trabajando desde esa estrategia, que toquen solamente los vientos y visualmente ya los tengo ahí y ellos saben que están ahí y se agrupan, entonces esa estrategia a mí me sirve muchísimo porque primero los niños viven una experiencia casi como de orquesta. (Docente 1)

Esto permite que el alumnado escuche a sus pares que están tocando las mismas melodías y puedan corregir o puedan notar cuándo se equivocan. El Docente 1 dice: "cuando tienes el mismo grupo instrumental, el flautista sabe cuándo está tocando una nota mal porque está escuchando al lado un mismo instrumento tocando otra cosa y ellos se dan cuenta". A esto, el Docente 2 agrega la necesidad de contar con una rutina de clase que permita normalizar el trabajo y las conductas del alumnado, en concreto, el Docente 2 precisa que "tener una rutina de clases es fundamental. Por ejemplo, saludan, crean una cantidad de compases para solfeo rítmico, escuchan una canción, luego cantan la canción". Asimismo, con el trabajo instrumental debe haber un orden al momento de abordar un nuevo arreglo, esto permite que el alumnado pueda identificar también los puntos esenciales para el trabajo. Específicamente, el Docente 2 indica que el primer punto tiene "que ver con escuchar, después ubicar las notas, solfeo hablado, después solfeo cantado, después tocar, después practicar y luego interpretar".

Dentro del montaje del arreglo van apareciendo diversas situaciones con las que hay que saber lidiar. Por ejemplo, alumnado que no quiera participar, estudiantes sin instrumentos... y ahí debe aparecer toda la flexibilidad para incluir al total del alumnado en el trabajo didáctico. El Docente 2 indica: "cuando hay un alumno que no está participando o lo ves medio extraño en la clase, hay que buscarle otra labor, tal vez probar con algún instrumento de percusión o que cante, pero mantenerlo ocupado con algo". Ahí se necesitan las herramientas del profesorado preparado, el Docente 2 añade," darle algún patrón rítmico más sencillo o más difícil, también puede ser el caso y esas ideas son múltiples e inagotables. Ahora esa idea de recombinar esas cosas proviene de tu aspecto de músico".

Una herramienta que debe estar entonces contemplada en el plan del trabajo del arreglo en Educación Primaria es la flexibilidad. Así, el Docente 2 indica: "Esa capacidad de flexibilizar es fundamental en un contexto..., si vas esperando que toquen todo puede que te caigas y para evitar eso hay que ir abiertos a desarmar la estructura melódica". Por consiguiente, el trabajo del arreglo en Educación Primaria requiere de una formación docente, ya que:

El profe de Música tiene que saber arreglar para educación primaria.... Creo que es un espacio en donde el profe tiene que ser muy bueno para trabajar. Los profes más grosos [mejores profesores] que yo he conocido, su mayor desafío ha sido trabajar con los más chicos; más que con los grandes. (Docente 2)

En consecuencia, la segunda categoría conceptual concerniente al arreglo como estrategia didáctica se comprende a partir de cinco códigos abiertos. A saber:

-La motivación docente y del estudiantado;

-Los componentes técnico-teóricos del arreglo musical;

@ Jean Huillipan Rain y Rolando Ángel-Alvarado. The content of this article is the sole responsibility of the authors. The Revista Electrónica de LEEME and Universitat de València are not liable for any legal actions that may arise involving the article's content. Revista Electrónica de LEEME - Lista Electrónica Europea de Música en la Educación-. http://ojs.uv.es/index/php/LEEME/index ISSN: 1575-9563. Editores: Universidad de Valencia y Jesús Tejada. Visibilidad de esta revista: SCOPUS, Emerging Sources Citation Index (Clarivate), EBSCO, CINDOC (CSIC), Citefactor, COPAC, Dialnet, DICE (CSIC), DOAJ, e-revistas (CSIC), EBSCO Premier, ERIH+, Gale Cengage Learning, IN-RECS, IRESIE, LATINDEX, MIAR, OCLC Worldcat, RESH, REDIB, RILM Core Journals, SUDOC, ULRICHS. Esta revista es de acceso libre mediante licencia Creative Commons 4.0 CC by. Política de archivado: etiqueta verde SHERPA-ROMEO. 
- La estructura del arreglo;

-La organización en el aula y;

- La idoneidad docente.

\section{Discusión y conclusiones}

Considerando el currículo nacional chileno para la Educación Primaria (MINEDUC, 2013), el profesorado enfatizó que el repertorio sugerido por dichas políticas educativas no es atingente a la realidad escolar porque plantean un desfase temporal, dado que son obras antiguas. A pesar de que las sugerencias didácticas del MINEDUC no representan un estímulo para docentes y discentes, la motivación del profesorado permite la realización del arreglo ya que actúa bajo sus ideas, deseos, metas y especialmente su propia voluntad (Carrillo, Padilla, Rosero y Villagómez, 2009). En vista de que el repertorio propuesto por el currículo nacional no es llamativo, cabe destacar que es el profesorado quien propone los arreglos del repertorio para trabajar en el aula, bajo el supuesto de que el currículo debe ser abordado y deliberado por ellos, según la realidad en la que se encuentren. De esta manera, se posibilita la interacción de la práctica musical, el crecimiento intelectual y la integración al contexto sociomusical del estudiantado (Ángel-Alvarado, 2020).

En cuanto al arreglo como estrategia didáctica se observaron tres aspectos fundamentales para el desarrollo de esta estrategia (Figura 3). Primero, la capacidad docente para flexibilizar a la hora de diseñar adecuaciones didácticas, tanto en los aspectos teóricomusicales del arreglo en sí, como en la resolución de situaciones de aula para priorizar el trabajo participativo e inclusivo en la actividad. Por lo tanto, el docente necesita una cualificación que no se limita solo al conocimiento musical (Lorenzo 2018), sino que se debe extender hacia lo pedagógico, dado que "el profesorado debe tener las competencias musicales para proporcionar una educación de calidad y las competencias profesionales que le permitan generar un clima propicio en el aula para hacer partícipe al estudiantado en la materia” (Carrillo y Vilar, 2014). Esto permitirá que el profesorado pueda ser capaz de afrontar las diversas situaciones del aula con éxito.

@ Jean Huillipan Rain y Rolando Ángel-Alvarado. The content of this article is the sole responsibility of the authors. The Revista Electrónica de LEEME and Universitat de València are not liable for any legal actions that may arise involving the article's content. Revista Electrónica de LEEME - Lista Electrónica Europea de Música en la Educación-. http://ojs.uv.es/index/php/LEEME/index ISSN: 1575-9563. Editores: Universidad de Valencia y Jesús Tejada. Visibilidad de esta revista: SCOPUS, Emerging Sources Citation Index (Clarivate), EBSCO, CINDOC (CSIC), Citefactor, COPAC, Dialnet, DICE (CSIC), DOAJ, e-revistas (CSIC), EBSCO Premier, ERIH+, Gale Cengage Learning, IN-RECS, IRESIE, LATINDEX, MIAR, OCLC Worldcat, RESH, REDIB, RILM Core Journals, SUDOC, ULRICHS. Esta revista es de acceso libre mediante licencia Creative Commons 4.0 CC by. Política de archivado: etiqueta verde SHERPA-ROMEO. 


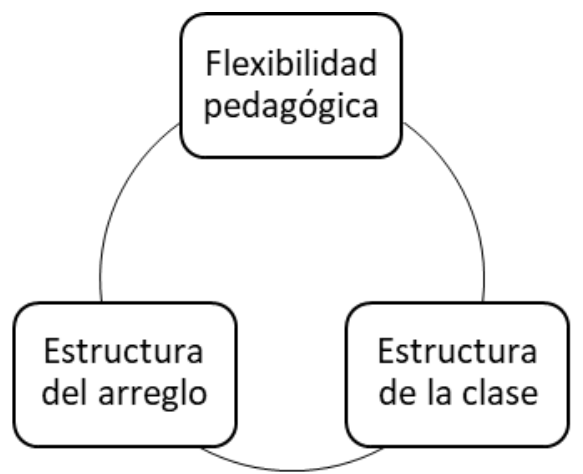

Figura 3. Tres aspectos fundamentales para el desarrollo del arreglo como estrategia didáctica Fuente: Elaboración propia

El segundo aspecto fundamental se refiere a la instauración de una estructura escolar que permita al estudiantado asimilar e interiorizar una rutina en el aula, centrándose en la creación de un ambiente propicio para el aprendizaje (Figura 3). En este punto, es conveniente también considerar la ubicación espacial de los instrumentos dentro del aula de clases, ya que su organización y orden por familia - vientos, percusiones, cuerdas - permite generar un espacio que garantiza el proceso de adquisición y desarrollo de los aprendizajes, las relaciones interpersonales y la socialización de experiencias, tras brindarse un apoyo contextualizado (Ángel-Alvarado, Belletich y Wilhelmi, 2019a).

Por último, el tercer aspecto fundamental se fija en la estructura del arreglo como un elemento con partes constitutivas claras (Figura 3). Por ejemplo, una introducción, estrofa e interludio. En este caso, cabe destacar que un docente participante enfatizó en la importancia de que el estudiantado tome consciencia de la estructura composicional de los arreglos, de cómo abordarlos y como ser participantes activos en la actividad. Para ello, es fundamental promover una pedagogía dialogante (De Zubiría, 2006) donde el estudiantado pueda emitir opiniones sobre el ensayo y la interpretación, así como también sugerir propuestas en algunas secciones musicales que presenten complejidad, asumiendo un rol activo durante los procesos de adquisición de aprendizajes y su desarrollo (Lorenzo, 2014).

A raíz de estos aspectos fundamentales se han podido identificar tres factores pedagógicos que influyen en el montaje de arreglos musicales en el aula regular de Educación Primaria (Figura 4). Primero, la autonomía pedagógica para tomar decisiones y deliberar sobre la selección, composición y montaje de los arreglos musicales. Esta deliberación sucede porque el docente conoce el contexto donde se encuentra inserto, tiene vínculos pedagógicos con la comunidad educativa que le otorgan la posibilidad de decidir en dicha materia, siempre en bienestar del crecimiento significativo esperado, aportando así a la ampliación cultural y social del estudiantado.

@ Jean Huillipan Rain y Rolando Ángel-Alvarado. The content of this article is the sole responsibility of the authors. The Revista Electrónica de LEEME and Universitat de València are not liable for any legal actions that may arise involving the article's content. Revista Electrónica de LEEME - Lista Electrónica Europea de Música en la Educación-. http://ojs.uv.es/index/php/LEEME/index ISSN: 1575-9563. Editores: Universidad de Valencia y Jesús Tejada. Visibilidad de esta revista: SCOPUS, Emerging Sources Citation Index (Clarivate), EBSCO, CINDOC (CSIC), Citefactor, COPAC, Dialnet, DICE (CSIC), DOAJ, e-revistas (CSIC), EBSCO Premier, ERIH+, Gale Cengage Learning, IN-RECS, IRESIE, LATINDEX, MIAR, OCLC Worldcat, RESH, REDIB, RILM Core Journals, SUDOC, ULRICHS. Esta revista es de acceso libre mediante licencia Creative Commons 4.0 CC by. Política de archivado: etiqueta verde SHERPA-ROMEO. 


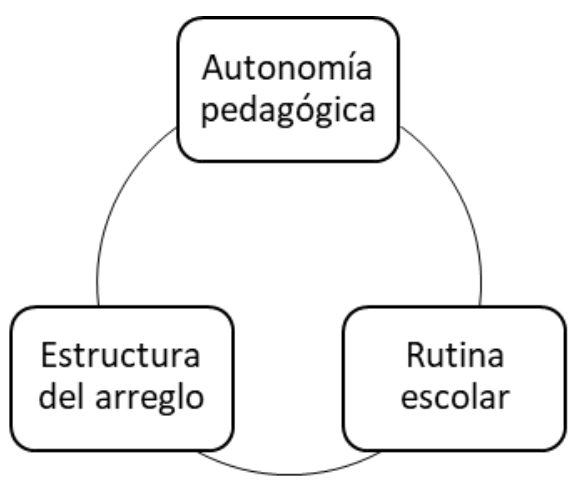

Figura 4. Tres factores pedagógicos que influyen al montar arreglos musicales en el aula regular Fuente: Elaboración propia

La segunda estrategia se basa en la relevancia de implantar una rutina escolar basada en hábitos de orden en todos los aspectos (Figura 4). Por ejemplo, organizar el aula para la práctica musical, instalar una rutina de actividades didácticas, distribuir los instrumentos musicales en el espacio didáctico, promover el cuidado de los instrumentos y del resto de insumos que existen en el aula. En definitiva, la internalización de estos hábitos hace plausible que el estudiantado experimente actividades musicales de mayor calidad en el contexto escolar.

Finalmente, la importancia de brindar aprendizajes contextualizados que permitan interiorizar la estructura de los arreglos ya sea en cuestiones de forma musical, así como también de los tiempos en que corresponde tocar o cantar (Figura 4). Durante la Educación Primaria, es menester de los estudiantes concentrarse en una sola actividad, de modo que se debe evitar al máximo que toquen y canten a la vez en la misma sección del arreglo. Ambas acciones siempre se deben llevar a cabo en distintos momentos. Únicamente se recomienda la práctica conjunta de tocar y cantar si los instrumentos que utilizan son de percusión y esto se debiera realizar en primera instancia para aprender el pulso y algunos ostinatos rítmicos.

A la vista de los factores pedagógicos identificados surgen tres implicaciones. Primero, desde la dimensión teórica, es necesario replicar el estudio, poniendo el foco en cada uno de los niveles educativos para obtener información más específica de las estrategias, materiales y tipos de arreglo que se pueden abordar en los distintos niveles. Segundo, desde la dimensión práctica, es imperioso que la administración escolar otorgue a la comunidad un espacio físico óptimo, implementado y adaptado para llevar a cabo las clases de Música. Que se provea al profesorado de perfeccionamiento en los ámbitos tanto musicales como pedagógicos para lograr mejorar las prácticas pedagógicas en lo que respecta a los arreglos musicales. Por último, desde la dimensión pedagógica, es esencial que el profesorado busque espacios de perfeccionamiento pedagógico-musical, así como también genere redes con otros docentes con el ánimo de organizar actividades conjuntas como festivales o conciertos.

@ Jean Huillipan Rain y Rolando Ángel-Alvarado. The content of this article is the sole responsibility of the authors. The Revista Electrónica de LEEME and Universitat de València are not liable for any legal actions that may arise involving the article's content. Revista Electrónica de LEEME - Lista Electrónica Europea de Música en la Educación-. http://ojs.uv.es/index/php/LEEME/index ISSN: 1575-9563. Editores: Universidad de Valencia y Jesús Tejada. Visibilidad de esta revista: SCOPUS, Emerging Sources Citation Index (Clarivate), EBSCO, CINDOC (CSIC), Citefactor, COPAC, Dialnet, DICE (CSIC), DOAJ, e-revistas (CSIC), EBSCO Premier, ERIH+, Gale Cengage Learning, IN-RECS, IRESIE, LATINDEX, MIAR, OCLC Worldcat, RESH, REDIB, RILM Core Journals, SUDOC, ULRICHS. Esta revista es de acceso libre mediante licencia Creative Commons 4.0 CC by. Política de archivado: etiqueta verde SHERPA-ROMEO. 
Si bien el presente estudio se contextualiza en la realidad chilena, sus hallazgos e implicaciones pueden servir a la comunidad iberoamericana porque articulan los saberes musicales que conlleva un arreglo con los saberes pedagógicos que implica un montaje musical en el aula escolar. En definitiva, el arreglo no se puede entender simplemente como música, sino que debe comprenderse como una instancia de aprendizaje y desarrollo.

\section{Referencias}

Ángel-Alvarado, R. (2018). Controversias entre la teoría curricular y la práctica educativa en la educación musical. Revista Electrónica Complutense de Investigación en Educación Musical, 15, 83-95. doi: 10.5209/RECIEM.58530

Ángel-Alvarado, R. (2020). The Crisis in Music Education Resulting from the Demise of Educational Institutions. Revista Educación, 44(1), 1-14. doi: 10.15517/revedu.v44il.39188

Ángel-Alvarado, R., Belletich, O. y Wilhelmi, M.R. (2019a). Motivación del estudiantado de secundaria en actividades de paisaje sonoro: Un estudio cuasiexperimental en un contexto de vulnerabilidad social. Revista Electrónica Educare, 23(2), 1-17. doi: $10.153950 /$ ree. $23-2.18$

Ángel-Alvarado, R., Wilhelmi, M.R. y Belletich, O. (2019b). Holistic Architecture for Music Education: A research design for carrying out empiric and interdisciplinary studies in didactics of music. Itamar, Revista de Investigación Musical: Territorios para el Arte, 5, 335-337. Recuperado de: http://ojs.uv.es/index.php/ITAMAR/article/view/15828/14281

Bonilla-García, M. y López-Suárez, A. (2016). Ejemplificación del proceso metodológico de la teoría fundamentada. Revista de Epistemología de las Ciencias Sociales, 57, 305-315. doi:10.4067/S0717-554X2016000300006

Cantón, I., y Vargas, G., (2014). Del currículum musical prescrito al currículum musical práctico en el aula de educación primaria. Bordón. Revista de Pedagogía, 62(2), 109126. Recuperado de: http://recyt.fecyt.es/index.php/BORDON/article/view/29184

Carey, G., Harrison, S. y Dwyer, R. (2017). Encouraging reflective practice in conservatoire students: A pathway to autonomous learning? Music Education Research, 19(1), 99110. doi:10.1080/14613808.2016.1238060

Carrillo, M., Padilla, J., Rosero, T. y Villagómez, M. (2009). La motivación y el aprendizaje. Alteridad, 4(2), 20-23. doi: 10.17163/alt.v4n2.2009.03

@ Jean Huillipan Rain y Rolando Ángel-Alvarado. The content of this article is the sole responsibility of the authors. The Revista Electrónica de LEEME and Universitat de València are not liable for any legal actions that may arise involving the article's content. Revista Electrónica de LEEME - Lista Electrónica Europea de Música en la Educación-. http://ojs.uv.es/index/php/LEEME/index ISSN: 1575-9563. Editores: Universidad de Valencia y Jesús Tejada. Visibilidad de esta revista: SCOPUS, Emerging Sources Citation Index (Clarivate), EBSCO, CINDOC (CSIC), Citefactor, COPAC, Dialnet, DICE (CSIC), DOAJ, e-revistas (CSIC), EBSCO Premier, ERIH+, Gale Cengage Learning, IN-RECS, IRESIE, LATINDEX, MIAR, OCLC Worldcat, RESH, REDIB, RILM Core Journals, SUDOC, ULRICHS. Esta revista es de acceso libre mediante licencia Creative Commons 4.0 CC by. Política de archivado: etiqueta verde SHERPA-ROMEO. 
Carrillo, C. y Vilar, M. (2014). El perfil profesional del profesorado de música: una propuesta de las competencias deseables en Ed. Primaria y Ed. Secundaria. Lista Electrónica Europea de LEEME, 33, 1-26. Recuperado de: http://ojs.uv.es/index.php/LEEME/article/view/9856

De Zubiría, J. (2006). Los modelos pedagógicos: Hacia una pedagogía dialogante. Bogotá: Cooperativa Editorial Magisterio.

Elliott, D.J. y Silverman, M. (2015). Music matters: A philosophy of music education. Nueva York, NY: Oxford University Press.

Fautley, M. (2017). Notation and music education. British Journal of Music Education, 34(2), 123-126. doi:10.1017/S0265051717000031

Kertz-Welzel, A. (2018). Globalizing music education: A framework. Bloomington, Indiana: Indiana University Press.

López-Iñiguez, G. y Pozo, J.I. (2014a). Like teacher, like student? Conceptions of children from traditional and constructive teachers regarding the teaching and learning of string instruments. Cognition and Instruction, 32(3), 219-252. doi:10.1080/07370008.2014.918132

López-Iñiguez, G. y Pozo, J. I. (2014b). The influence of teachers' conceptions on their students' learning: children's understanding of sheet music. British Journal of Educational Psychology, 84(2), 311-328. doi:10.1111/bjep.12026

Lorenzo, M. (2018). La docencia de la música de conjunto en las Escuelas de Música. En busca de soluciones a su problemática dentro y fuera del aula. Artseduca, 20, 44-67. doi:10.6035/Artseduca.2018.20.3

Ministerio de Educación (2013). Programa de estudio cuarto año básico. Santiago de Chile, MINEDUC.

Muñoz, M., López, M. y Assaél, J. (2015). Concepciones docentes para responder a la diversidad: ¿Barreras o recursos para la inclusión educativa? Psicoperspectivas, 14(3), 68-79 DOI:10.5027/psicoperspectivas-Vol14-Issue3-fulltext-646

Odendaal, A., Kankkunen, O-T., Nikkanen, H.M. y Vakeva, L. (2014). What's with the K? Exploring the implications of Christopher Small's 'musicking' for general music education. Music Education Research, 16(2), 162-175. doi:10.1080/14613808.2013.859661

@ Jean Huillipan Rain y Rolando Ángel-Alvarado. The content of this article is the sole responsibility of the authors. The Revista Electrónica de LEEME and Universitat de València are not liable for any legal actions that may arise involving the article's content. Revista Electrónica de LEEME - Lista Electrónica Europea de Música en la Educación-. http://ojs.uv.es/index/php/LEEME/index ISSN: 1575-9563. Editores: Universidad de Valencia y Jesús Tejada. Visibilidad de esta revista: SCOPUS, Emerging Sources Citation Index (Clarivate), EBSCO, CINDOC (CSIC), Citefactor, COPAC, Dialnet, DICE (CSIC), DOAJ, e-revistas (CSIC), EBSCO Premier, ERIH+, Gale Cengage Learning, IN-RECS, IRESIE, LATINDEX, MIAR, OCLC Worldcat, RESH, REDIB, RILM Core Journals, SUDOC, ULRICHS. Esta revista es de acceso libre mediante licencia Creative Commons 4.0 CC by. Política de archivado: etiqueta verde SHERPA-ROMEO. 
San Martín, D. (2014). Teoría fundamentada y Atlas.ti: Recursos metodológicos para la investigación educativa. Revista Electrónica de Investigación Educativa, 16(1), 104122.

Salvador, K. (2019). Assessment and individualized instruction in elementary general music: A case study. Research Studies in Music Education, 41(1), 18-42. doi:10.1177/1321103X18773092

Silva, R. (2019). La ópera como herramienta de acción social y unión comunitaria. Reflexiones acerca del proceso creativo, montaje y estreno de La Malén. Revista Musical Chilena, 73(231), 147-155. doi:10.4067/S0716-27902019000100147

@ Jean Huillipan Rain y Rolando Ángel-Alvarado. The content of this article is the sole responsibility of the authors. The Revista Electrónica de LEEME and Universitat de València are not liable for any legal actions that may arise involving the article's content. Revista Electrónica de LEEME - Lista Electrónica Europea de Música en la Educación-. http://ojs.uv.es/index/php/LEEME/index ISSN: 1575-9563. Editores: Universidad de Valencia y Jesús Tejada. Visibilidad de esta revista: SCOPUS, Emerging Sources Citation Index (Clarivate), EBSCO, CINDOC (CSIC), Citefactor, COPAC, Dialnet, DICE (CSIC), DOAJ, e-revistas (CSIC), EBSCO Premier, ERIH+, Gale Cengage Learning, IN-RECS, IRESIE, LATINDEX, MIAR, OCLC Worldcat, RESH, REDIB, RILM Core Journals, SUDOC, ULRICHS. Esta revista es de acceso libre mediante licencia Creative Commons 4.0 CC by. Política de archivado: etiqueta verde SHERPA-ROMEO. 